\title{
THE BLOOD FLOW IN THE BRAIN AND THE LEG OF MAN, AND THE CHANGES INDUCED BY ALTERATION OF BLOOD GASES ${ }^{1}$
}

\author{
By WILLIAM G. LENNOX AND ERNA LEONHARDT GIBBS \\ (From the Department of Neuropathology, Harvard Medical School, and the Thorndike \\ Laboratory of the Boston City Hospital, Boston)
}

(Received for publication July 5, 1932)

The solution of various neurological problems awaits an increase in our knowledge of the circulation of blood within the brain. Much has been gained by the demonstration of Forbes (1) and his co-workers that the pial vessels of the cat are under both vasomotor and chemical control. These experiments consisted of actual inspection and measurement of pial vessels viewed through a window screwed in the skull, the pressure of the cerebrospinal fluid and of the blood being measured simultaneously. In particular, Wolff and Lennox (2) showed that changes in the gaseous content of the blood had a prompt and clear cut effect on the diameter of pial vessels, an effect which was independent of the systemic blood pressure. These authors observed that an increase in the carbon dioxide content of the blood was attended by an extreme dilatation of pial vessels whereas a decrease was followed by a constriction. Variations in oxygen content resulted in relatively small changes. An increase in oxygen tension produced a slight constriction and a decrease in tension a slight dilatation of the observed vessels.

There are two difficulties in the way of accepting these observations as significant of cerebral circulatory changes in man. First, experiments were conducted on cats under amytal anesthesia. Second, the vessels lying in the pial covering of the brain may act differently from vessels lying within the brain. Intracranial circulation is of such great importance to the organism and is so modified by the closed box arrangement of the skull that there is need for specific experimentation in man.

Observations concerning blood flow through the brain of unanesthetized persons seemed impossible until Myerson, Halloran and Hirsch (3) demonstrated the feasibility of obtaining blood from the internal jugular vein. By means of this procedure, we have repeated the experimental observations of Wolff and Lennox (2), using patients and judging

1 This is paper XX in the series of papers on "The Cerebral Circulation" from the Department of Neuropathology, Harvard Medical School. It is also from the Thorndike Laboratory of the Boston City Hospital. Work was done under a grant from the Harvard Epilepsy Commission. 
changes in circulation by alterations in the gaseous content of blood entering and leaving the brain. In addition we compared the brain changes with those taking place in the leg.

A change in the oxygen content of venous blood during an experimental period might be indicative of alterations either in the oxygen consumption of the tissue traversed, or in the speed of the blood flowing through the tissues. The oxygen consumption of tissues is not affected by the tension of oxygen in the blood, within limits which were not overstepped in these experiments. Patients lay quietly throughout the period of observation, so that there was probably no great variation in the total metabolism. It is therefore believed that observed changes in arteriovenous difference were due to alterations in the speed of blood flow. Such change in pace might be consequent on change in heart rate and blood pressure, on variations in the number of open capillaries or on alterations in the size of arterioles. The observed changes in A-V difference did not parallel changes in systemic blood pressure or heart rate. We believe alterations in the size of the vascular bed is the correct explanation. This view is supported by animal experiments of Wolff and Lennox (2) in which pial vessels were watched under the microscope and by those of Bronk and Gesell (4) in which the volume of blood perfusing the head and leg was measured by means of thermopiles. Both these experimenters observed that blood flow through the parts studied did not depend on blood pressure. We believe that decreases in $\mathrm{A}-\mathrm{V}$ difference (in which venous blood becomes more arterial-like) are due in our experiments to a dilatation of the arteriolar bed, leading to an increased speed and volume of blood flow. Increases in $\mathrm{A}-\mathrm{V}$ differences (in which venous blood becomes more venous) are due to a constriction of the arteriolar bed with a slowing of the blood stream.

\section{MATERIAL AND METHODS}

The subjects used in this study were neurological patients at the Boston City Hospital, for the most part epileptics. None had serious cardiac or pulmonary defect. Observations were made only with those patients who expressed a willingness to cooperate in observations designed to increase knowledge concerning their intracranial circulation.

Observations were made in the morning, patients having fasted since the previous evening and having lain quietly for at least half an hour. Blood was taken without stasis (after anesthetization of the overlying skin) under oil and was immediately chilled and analyzed in the portable Van Slyke apparatus (5). Duplicate measurements were made and differed no more than one-tenth of a volume per cent. Arterial blood was taken from a brachial, radial or femoral artery. Blood from the internal jugular vein was secured by the method described by Myerson and associates (3), except that a small needle ( $1 \frac{1}{2}$ inch 20 gauge) was used and the head was kept in a comfortable position in a line horizontal with the rest of the body.

In previous observations with the same procedure, such as those with histamine by Weiss and Lennox (6), a major difficulty had been the simultaneous 
taking of blood from the various vessels. In order to meet this difficulty, as well as the objection of repeated jugular punctures, we allowed the needles to remain in situ in the internal jugular and femoral veins during the minutes intervening between the drawing of the preliminary and the experimental bloods. A stylette was inserted in the needle to prevent leakage and clotting. Blood was drawn simultaneously from the two veins, followed by puncture of the artery.

For purpose of comparison with the brain, a leg rather than an arm was used. Blood from the femoral vein has passed through a relatively large proportion of muscle and the circulation of the leg is not so readily affected by changes in temperature.

In order to alter the gaseous content of the blood, variations in the composition of the alveolar air were induced. The procedures used will be described later. Because there was considerable variation in individual cases, observations of each experimental procedure were made from three to ten times. Individual measurements in each of the 50 experiments are shown in the accompanying Table I.

\section{RESULTS}

In order to distinguish the effects due to oxygen from those due to carbon dioxide, it was necessary to cover all the possible combinations of these gases. Consequently observations were separated into nine groups, with reference to whether the tension of oxygen or of carbon dioxide was increased, normal or decreased. A first additional condition was the normal control.

\section{Normal carbon dioxide-normal oxygen}

It is important to know the amount of variation in the concentration of the gases in blood drawn from vessels at intervals. In the control experiments, 15-20 minutes elapsed between the first and second blood samples. The average differences between successive samples (which are slight) are shown in Figure 1.

\section{Increased carbon dioxide and oxygen}

In five instances, patients breathed a mixture of 90 per cent oxygen and 10 per cent carbon dioxide. Interest attaches to these results because of the wide clinical use of a mixture of oxygen and carbon dioxide for the treatment of asphyxia, carbon monoxide poisoning and pneumonia. The gas was administered from a compressed air tank by means of a face mask. Some patients did not receive the full concentration because such violent hyperpnea was produced that dilution of the gas with air was necessary. The mixture was breathed for 5-10 minutes before blood samples were taken.

As a result of breathing this mixture the carbon dioxide content of arterial blood was increased by 3.7 volumes per cent, and the oxygen content by 0.9 volume per cent (Figure 2). The increase in the oxygen content was possible because two of the patients had an initially low arterial oxygen saturation. 


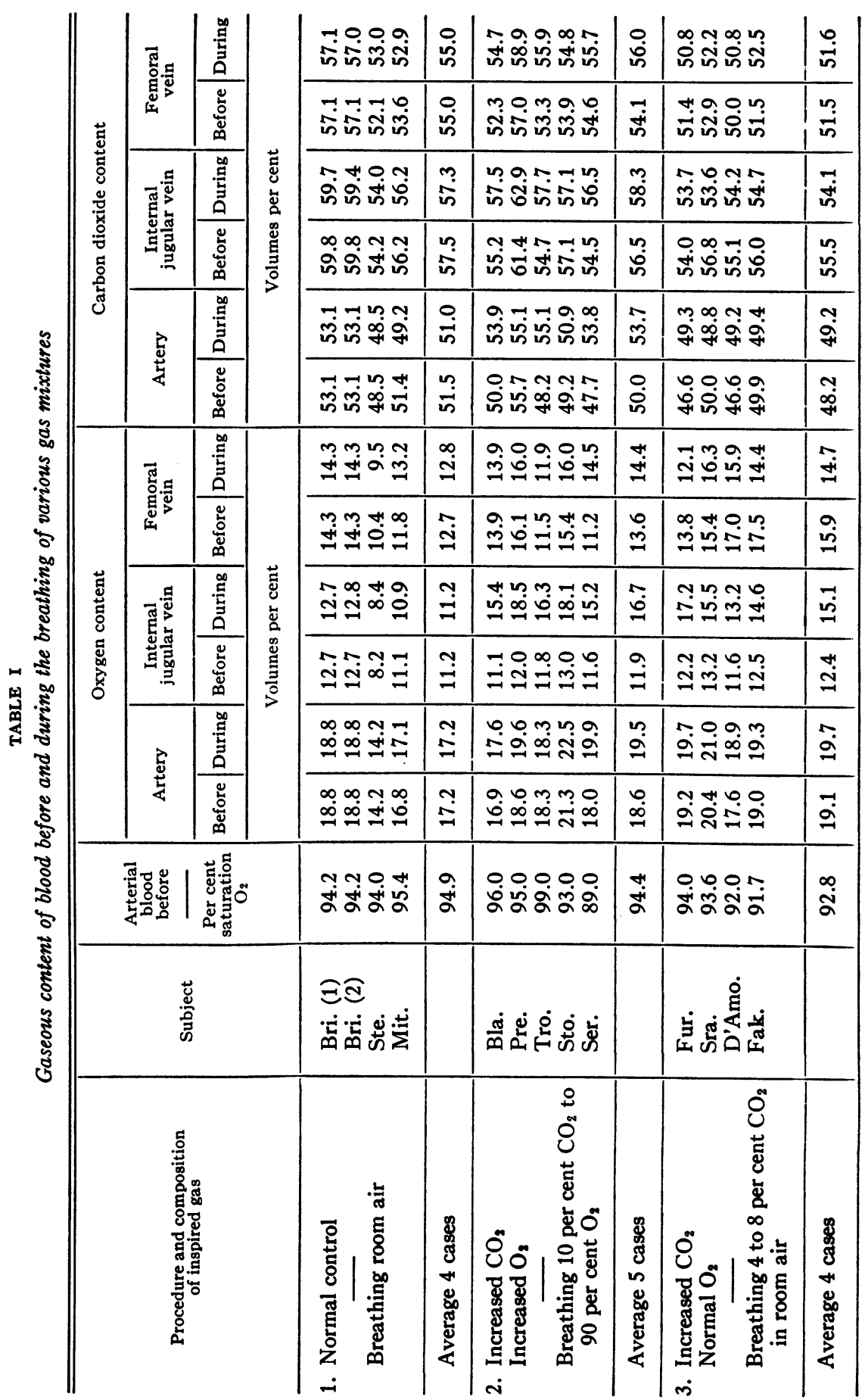




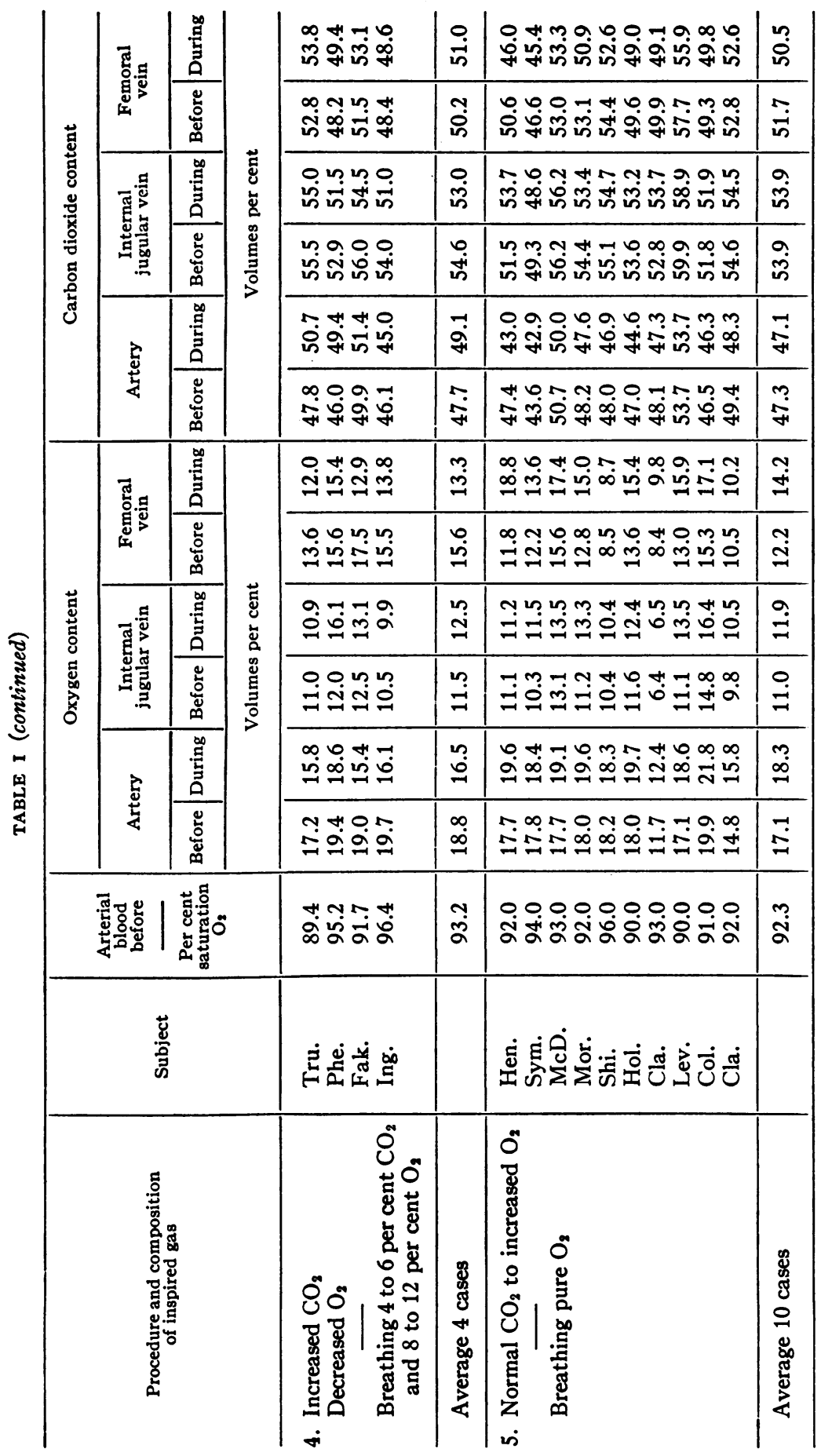




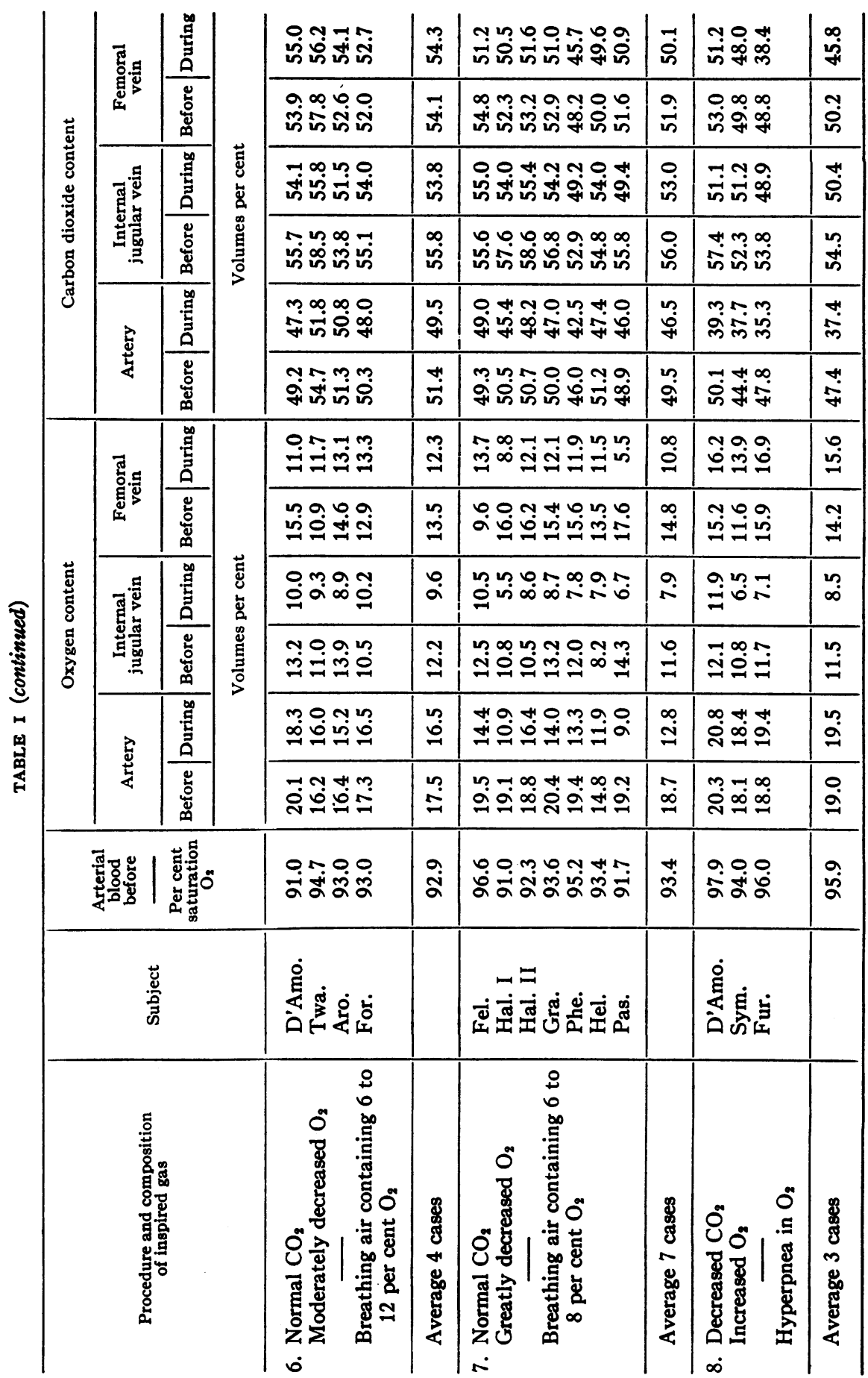




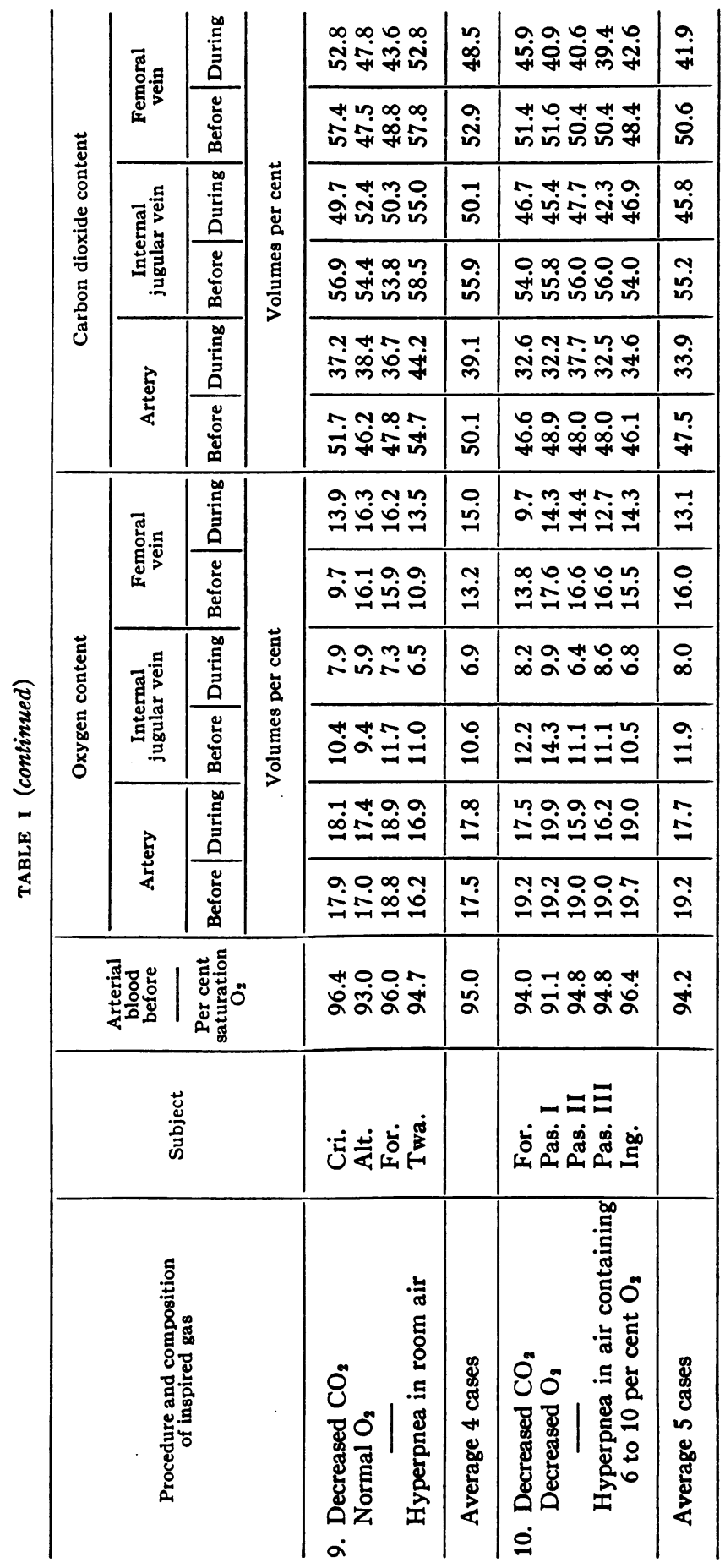


The effect on the intracranial circulation was striking. In all cases there was a great decrease in the $\mathrm{A}-\mathrm{V}$ difference, blood leaving the brain being nearly arterial, as regards its oxygen content. The average A-V difference was decreased from 6.7 volumes per cent to 2.8 volumes per cent, a decrease of 58 per cent. This indicated a vast acceleration of blood flow through the brain, a result which is in agreement with the animal experiments of Wolff and Lennox, which demonstrated a dilatation of pial vessels and an increase in spinal fluid pressure when cats breathed a

NORMAL $\mathrm{CO}_{2}$ - NORMAL $\mathrm{O}_{2}$

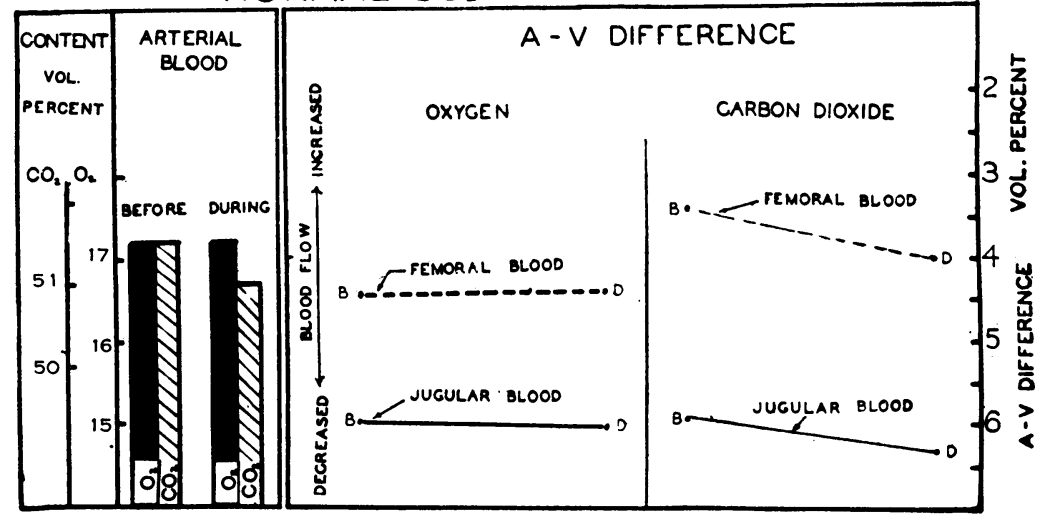

Fig. 1. Normal Control Group, Subjects Breathing Room Air

In this and all figures except the last, the columns in the left hand portion represent the oxygen and the carbon dioxide content of arterial blood before and during the experimental period. The curves in the right hand portion represent the alterations in the gaseous content of the blood during its passage through the tissues, i.e. the A-V difference before (B) and during (D) the procedure. These changes refer to both oxygen (heavy lines) and carbon dioxide (lighter lines). The A-V differences for the leg are represented by a dashed line and for the brain by a solid line. The steepness of the slant indicates the extent of the change. A line slanting downward from left to right means that there was increased $\mathrm{A}-\mathrm{V}$ difference during the experimental procedure and therefore a decreased speed of blood flow, due probably to a constriction of arterioles. An upward slant of the line represents the opposite condition. The various figures represent the average measurements of from three to 10 experiments.

similar mixture. There is agreement also with the human experiments of Cobb and Fremont-Smith (7) who noted that the color of retinal veins approached that of arteries, when subjects breathed a mixture of oxygen and carbon dioxide.

In surprising contrast to the behavior of cerebral vessels, the average A-V difference in the leg changed almost not at all (from 5.0 to 5.1 volumes per cent), an increase of two per cent. Individual results varied widely. This failure of the circulation in the leg to show changes similar to those in the brain is in keeping with the lack of a consistent rise or fall 
in blood pressure. If vessels all over the body were to dilate in response to increased arterial carbon dioxide as strongly as do the cerebral vessels, there would be a dramatic fall of blood pressure.

\section{Increased carbon dioxide-normal oxygen}

In these four experiments, a Tissot tank was filled with atmospheric air to which had been added sufficient carbon dioxide to make from four to eight per cent by volume, a concentration sufficient to induce intense hyperpnea, although as compared with the previous group of experiments, the gaseous content of the arterial blood was but little increased. There was an average increase of one volume per cent in the carbon dioxide and

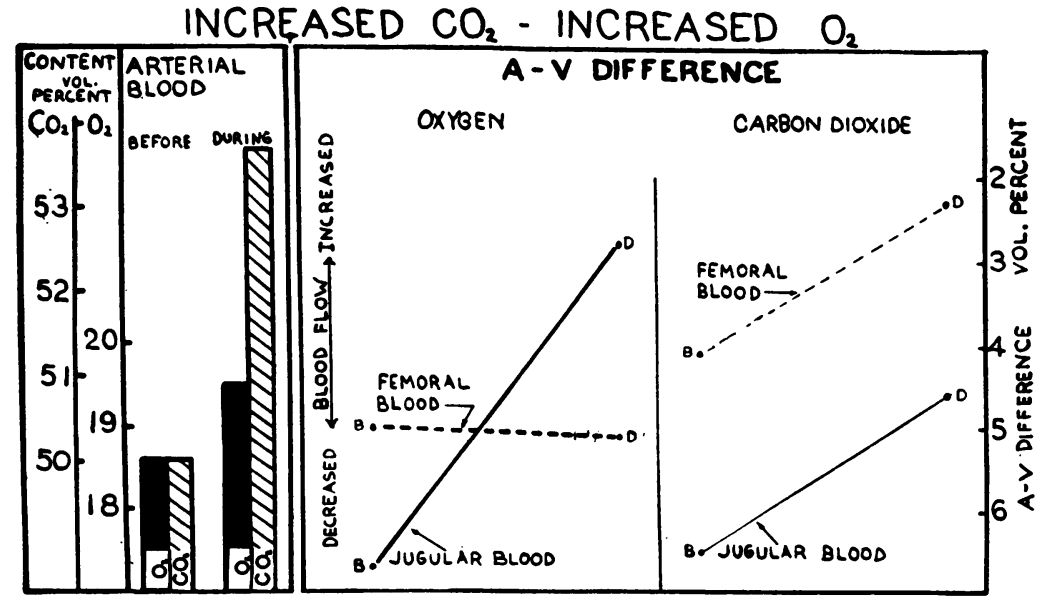

Fig. 2. Average Results in a Group of Five Patients Breathing Air Containing Approximately 10 Per Cent Carbon Dioxide and 90 Per Cent Oxygen.

The speed of blood flow through the brain is greatly increased and through the leg is not affected.

0.6 volume per cent in the oxygen content of the blood (Figure 3). Venous blood drawn after an interval of five or ten minutes showed that the A-V difference was decreased in the head by 31 per cent and was increased in the leg by 50 per cent, i.e. cerebral vessels had dilated and leg vessels had constricted. In both this and the preceding group, the results in each experiment were more consistent for the head than for the leg. Of the nine experiments, changes in $\mathrm{A}-\mathrm{V}$ difference in blood traversing the brain were all in the same direction, whereas in the leg, three were against the trend. The observed changes are in agreement with the animal experiments of Bronk and Gesell (4) who measured blood flow in the carotid and femoral arteries of dogs, and found the flow was increased in the carotid and decreased in the femoral artery when the animal breathed air rich in carbon dioxide. 


\section{Increased carbon dioxide-decreased oxygen}

In four instances, subjects breathed air from a Tissot tank to which both carbon dioxide and nitrogen had been added, to make a mixture containing from 4 to 6 per cent by volume of carbon dioxide and from 8 to 12 per cent by volume of oxygen. The average effect was to reduce the oxygen content of the arterial blood by 2.3 volumes per cent and to increase the carbon dioxide content by 1.4 volumes per cent (Figure 4). As in the two previous groups of experiments there was a great increase in the speed of blood flowing through the brain (a reduction in the $A-V$ difference of 7.3 volumes per cent; or 45 per cent) whereas the $A-V$ difference for the leg did not change.

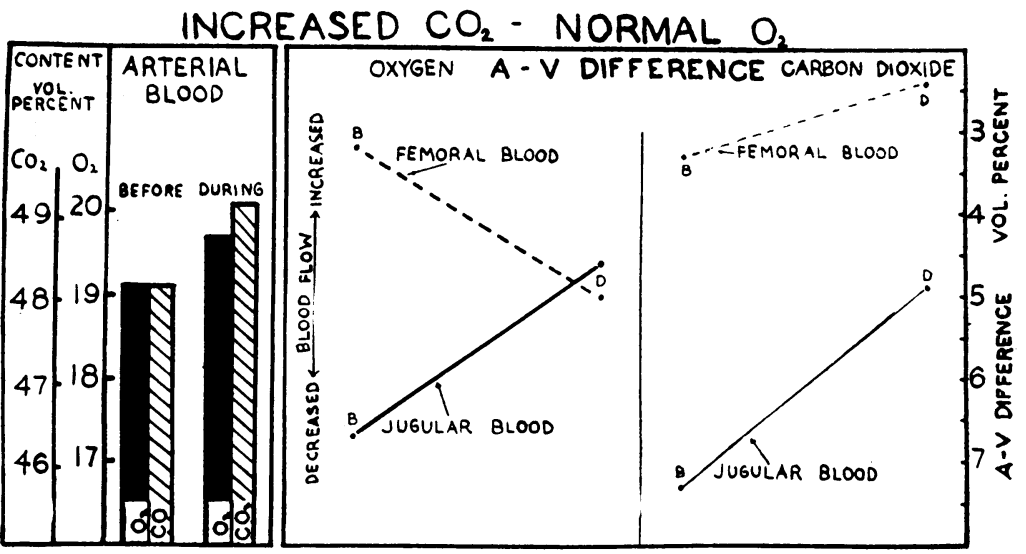

Fig. 3. Average Results in a Group of Four Subjects Breathing Room Air to Which Had Been Added from Four to Eight Per Cent by Volume of Carbon Dioxide.

The speed of blood flow through the brain was greatly increased and through the leg decreased.

In each of the foregoing experiments thirteen in all, in which carbon dioxide tension of arterial blood was increased, there was a consistent and striking decrease in the $\mathrm{A}-\mathrm{V}$ difference for the brain. The average decrease was 21 per cent for each volume per cent increase in the carbon dioxide content of arterial blood. In the individual cases, however, the degree of dilatation of cerebral vessels (as judged by decreases in A-V differences) was not proportional to the observed changes in the carbon dioxide content. As regards the leg, changes in A-V differences were not consistent. In eight instances there was an increase and in five a decrease.

\section{Normal carbon dioxide-increased oxygen}

In ten experiments, subjects were attached to a Collins-Roth metabolism apparatus and breathed pure oxygen for a period of from 10 to 15 
minutes. While breathing oxygen the carbon dioxide content of the arterial blood did not change appreciably but the average oxygen content increased by 1.2 volumes per cent (Figure 5). This was possible because the average arterial blood was but $\mathbf{9 2 . 3}$ per cent saturated. This condition of mild anoxemia in epileptic patients has been reported by Lennox (8). In contrast with the previous groups of experiments, changes in blood flow were slight and not consistent. For the brain, the A-V difference was increased in six instances and decreased in four, the average change being an increase of five per cent. For the leg, the A-V difference was increased in two instances and decreased in eight, the average change being a decrease of 16 per cent. The results therefore are indecisive, there

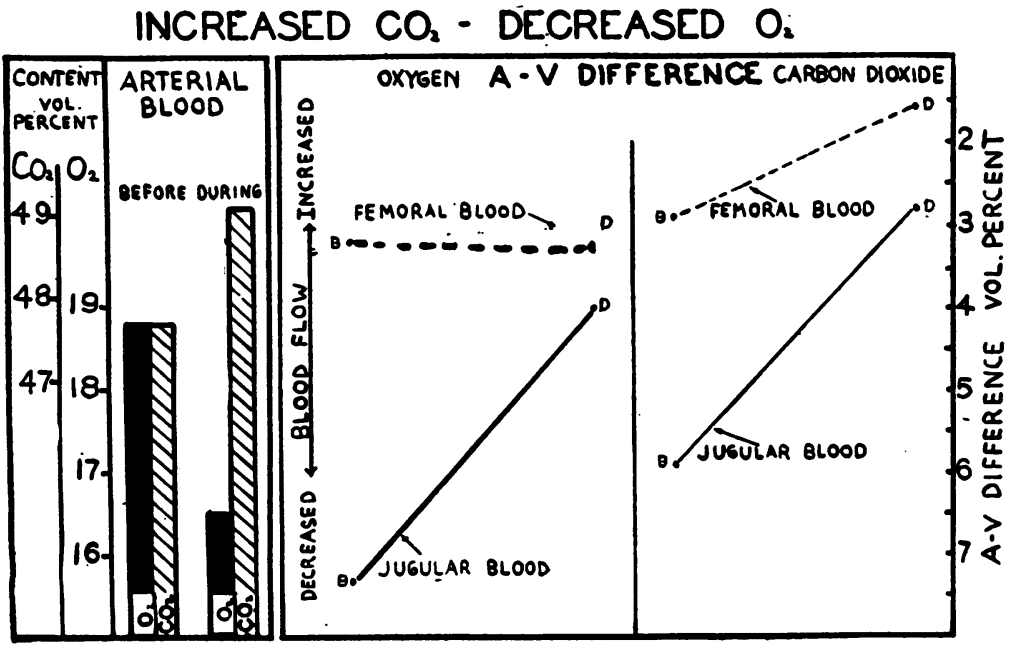

Fig. 4. Average Results in a Group of Four Subjects Who Breathed air Containing from Four to Six Per Cent by Volume of Carbon Dioxide and from 8 to 12 Per Cent of Oxygen.

The speed of blood flow through the brain was very greatly increased whereas the flow through the leg was not affected.

being evidence of a slight decrease in the speed of blood flow through the brain, and more consistent evidence of an increased flow through the leg. So many experiments were conducted because of the lack of consistency in results and because changes in circulation due to the breathing of oxygen have received scant attention in medical literature. In dog experiments Bernthal, Bronk, Cordero and Gesell (9) obtained evidence of a decreased blood flow in both the carotid and the femoral arteries. Our results for the circulation in the leg, therefore, are not in agreement with theirs. The difference may be due to the fact that presumably the anesthetized animals had a greater initial anoxemia than the human subjects. 


\section{Normal carbon dioxide-slightly decreased oxygen}

In four experiments subjects breathed from a Tissot tank to which atmospheric air and nitrogen had been added. Individuals breathing the same gas mixture showed large differences in the degree of anoxemia. Therefore, separation of cases was based on the actual degree of anoxemia present in arterial blood and not on the mixture breathed. The average reduction in arterial oxygen in the four subjects of this group was one volume per cent making the arterial blood 87 per cent saturated. Because of the hyperpnea induced by the diminished oxygen supply, the average carbon dioxide content of the arterial blood was decreased by 1.9 volumes per cent (Figure 6). In three of the four cases there was a sharp increase

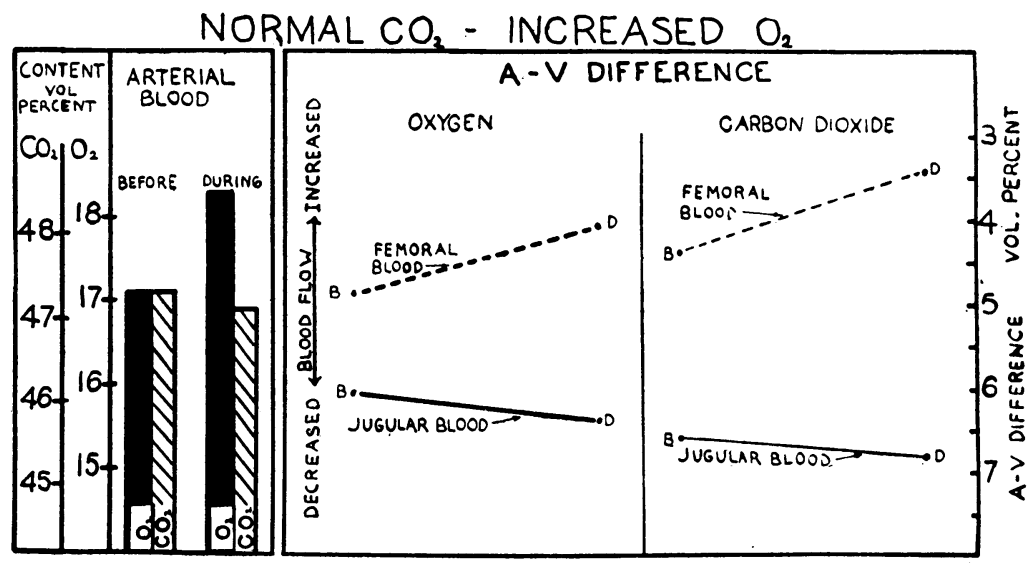

Fig. 5. Average Results in a Group of Ten Subjects Who Breathed Pure OXYgen

The speed of blood flow through the brain was slightly decreased, and through the leg slightly increased.

in the $\mathrm{A}-\mathrm{V}$ difference for the brain. The average increase for the four cases was 30 per cent. For the leg, the A-V difference was increased in two and decreased in two experiments, the average change being an increase of 5 per cent. The decreased speed of blood flow through the brain, in the light of evidence from other groups of experiments, was presumably caused by the reduction of carbon dioxide, rather than the reduction of oxygen in the arterial blood.

\section{Normal carbon dioxide-greatly decreased oxygen}

In these seven cases, a much greater degree of anoxemia was induced, the average oxygen content of arterial blood being reduced by 5.9 volumes per cent (from an average saturation of 93.4 per cent to 64 per cent). In spite of this anoxemia, the average carbon dioxide content of the arterial blood fell but three volumes per cent (Figure 7). 
In all seven instances there was a decrease in the difference in oxygen content between arterial and internal jugular blood, the average decrease being 31 per cent. In five instances there was likewise a great decrease in the difference between arterial and femoral vein blood (the average decrease for the seven cases being 48 per cent). Therefore, the vessels of both the brain and the leg underwent dilatation. This is in agreement with observations in the dog by Gesell and his associates. If all the blood vessels of the body dilated to this extent, there would be a fall in systemic blood pressure. We were not able to record the pulse and the blood pressure readings in all these cases, but in those in which we did, there was an increase in heart rate and a fall in blood pressure. According to Marshall (10) in conditions of anoxemia, cardiac output is increased only when the concentration of oxygen in the inspired air is less than 11

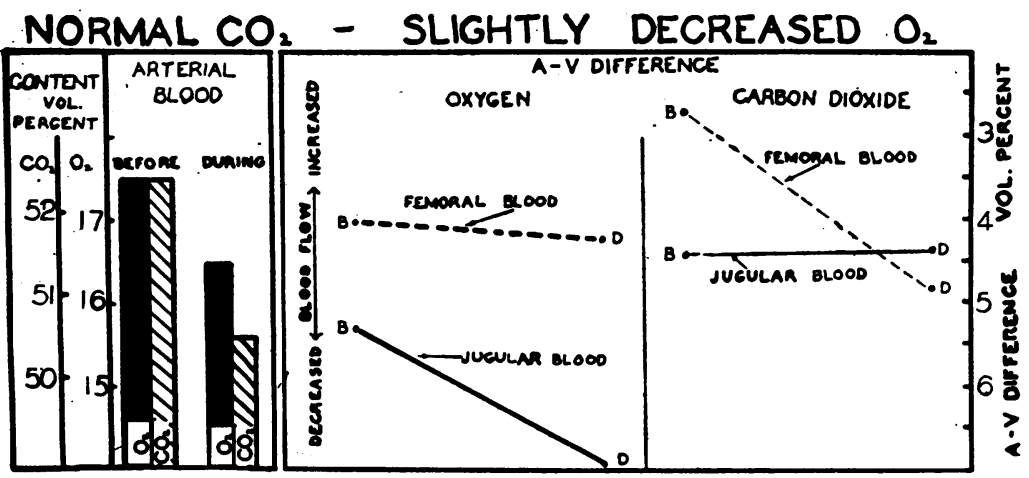

Fig. 6. Average Results in a Group of Four Subjects Who Breathed Air Containing from 6 to 12 Per Cent by Volume of Oxygen and Whose Arterial Oxygen Saturation Decreased from a Preliminary Value of 92.3 Per Cent to 87 Per Cent.

There was a moderate decrease in the speed of blood flow through the brain and a very slight decrease in the leg, due probably to the diminution in the tension of carbon dioxide in arterial blood.

per cent. The fact that there was decreased cerebral blood flow with slight anoxemia (Group 6) and increased flow with extreme anoxemia (Group 7) is probably explainable in this manner: The decreased carbon dioxide due to dyspnea exerted its constricting effect when anoxemia was trivial, but an even greater carbon dioxide loss could not prevent dilatation when anoxemia was extreme.

\section{Decreased carbon dioxide-increased oxygen}

In three experiments, subjects while connected with a basal metabolism machine and breathing pure oxygen, performed voluntary hyperpnea. Arterial blood drawn after five or ten minutes revealed an average increase of .5 volume per cent in oxygen content and a decrease of 10 volumes per cent in carbon dioxide content (Figure 8). 
In each instance there was an increase in the $\mathrm{A}-\mathrm{V}$ difference for the brain, the average increase being 47 per cent and a decrease for the leg, the average being 19 per cent. These changes indicate a decreased speed of blood flow through the brain and an increased flow through the leg.

\section{Decreased carbon dioxide-normal oxygen}

In this group of four experiments, the subjects performed overventilation in room air, a procedure which increased the average oxygen content of arterial blood .3 volume per cent and decreased its carbon

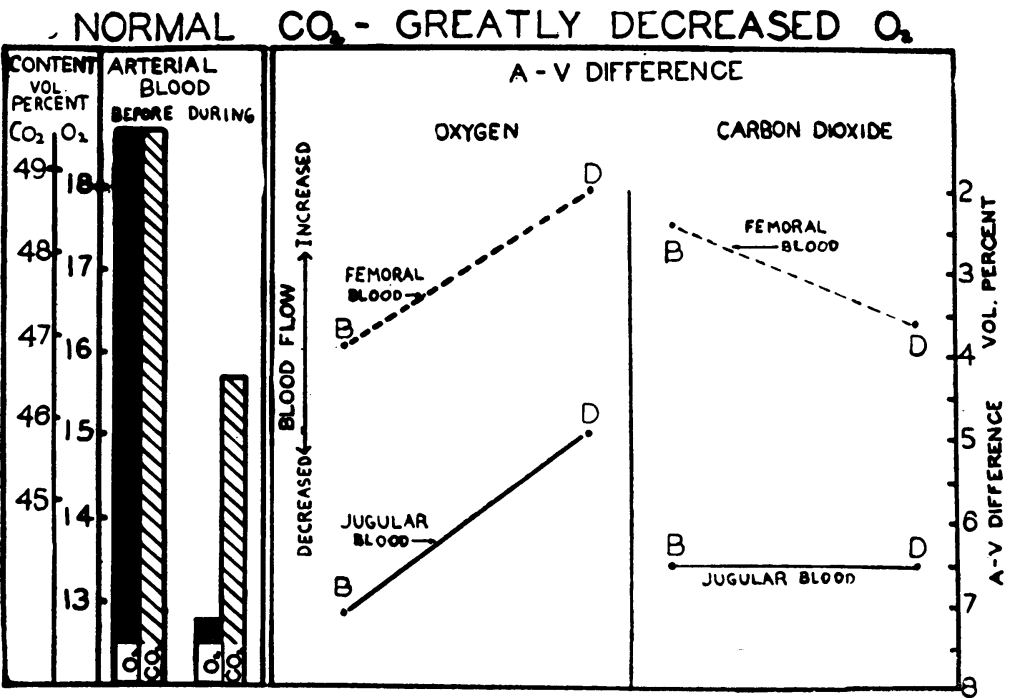

Fig. 7. Average Results in a Group of Seven Patients Who Breathed Air Containing from 6 to 8 Per Cent by Volume of Oxygen, and Whose Arterial Oxygen Saturation Decreased from a Preliminary Value of 93.4 Per Cent to 64 Per Cent.

The flow of blood through both the brain and the leg was greatly accelerated.

dioxide content by 11 volumes per cent. Results were not greatly different from those in the preceding group. The effect on blood flow was in the same direction though more distinct. For the brain there was an increase in the $\mathrm{A}-\mathrm{V}$ difference in all cases, the average increase being 58 per cent. For the leg there was a decrease in the A-V difference in three of the four cases, the average decrease for all being 35 per cent (Figure 9). The decrease in cerebral blood flow accompanying hyperpnea (per unit change in carbon dioxide content of arterial blood) is only one sixth as great as the increase which accompanies the breathing of carbon dioxide. 


\section{Decreased carbon dioxide-decreased oxygen}

In three experiments, subjects breathed a mixture of air and nitrogen (the oxygen forming from 6 to 10 per cent by volume of the mixture) from a Tissot tank, at the same time performing voluntary hyperpnea. In two experiments subjects were attached to a basal metabolism apparatus, the bell of which had been filled with room air. In this case anoxemia developed more slowly.

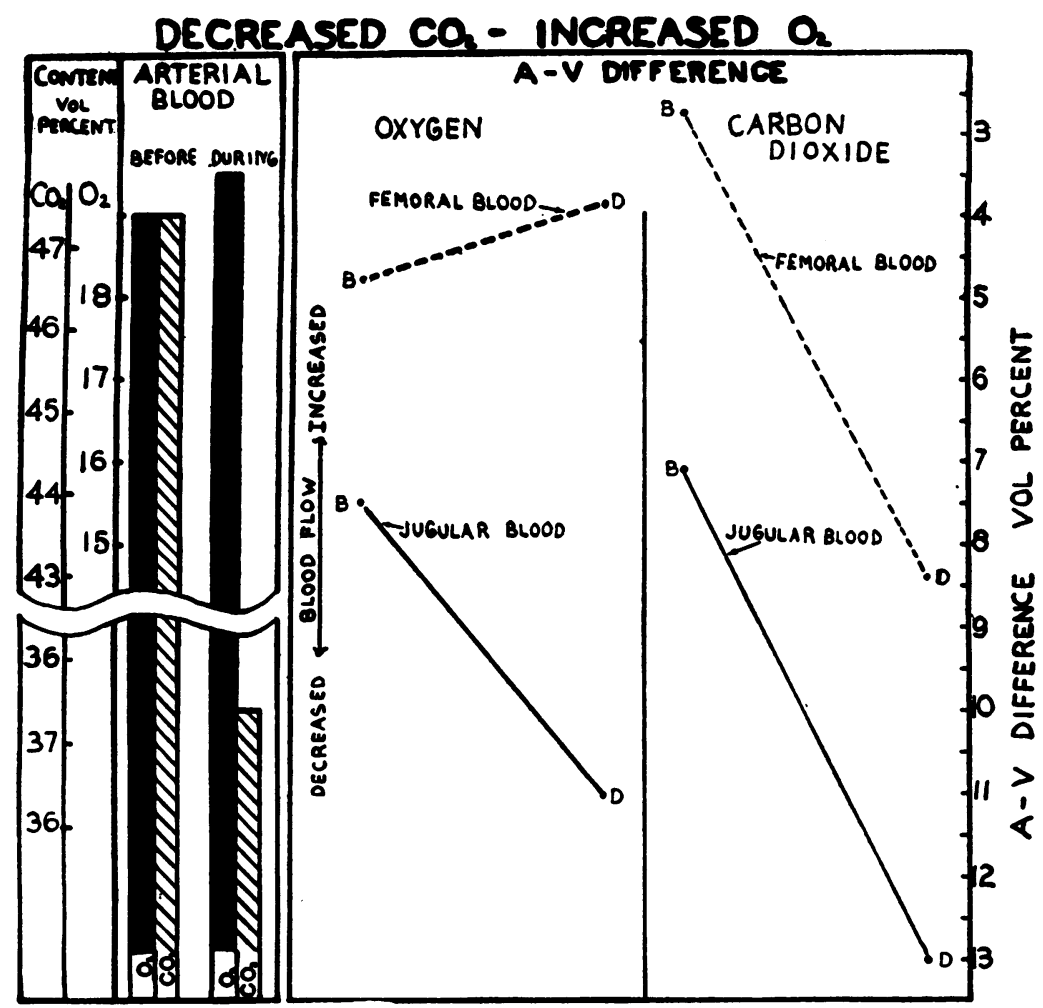

Fig. 8. Average Results in a Group of Three Subjects Who Performed Hyperpanea While Breathing Pure Oxygen

The speed of blood flow through the brain was greatly decreased and through the leg was slightly increased.

Although air mixtures were practically the same as in the experiments under Group 7, the anoxemia produced was not as great when subjects breathed deeply, as when they breathed naturally. The percentage saturation of arterial blood was reduced by only 6.6 per cent, against a reduction of 29.4 per cent when hyperpnea was not performed. Schneider (11) has stated that under conditions of reduced atmospheric pressure subjects are more comfortable if they breathe deeply. In the five experiments the average decrease in the oxygen content of arterial blood was 1.5 and of the carbon dioxide content 13.6 volumes per cent. 
In four instances, the A-V difference was increased both for the brain and the leg, in one it was decreased for both. For the five experiments, the average increase for the brain was 33 per cent and for the leg 44 per cent (Figure 10).

Although overbreathing in oxygen poor air resulted in a greater decrease in the carbon dioxide content of arterial blood than overbreathing in room air, the diminution in blood flow through the brain was less. Presumably this was due to the counteracting effect of the anoxemia which in itself would cause an increase in blood flow.

\section{DECREASED $\mathrm{CO}_{2}$ - NORMAL $\mathrm{O}_{2}$}

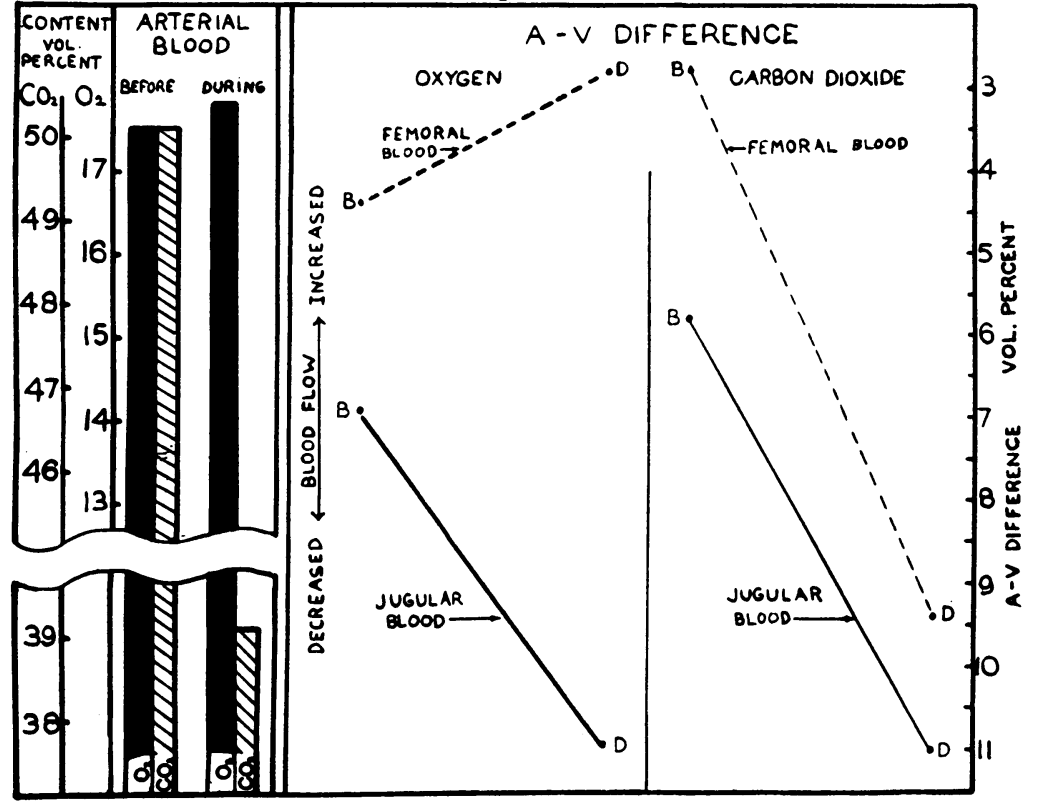

Fig. 9. Average Results in a Group of Four Subjects Who Performed Hyperpanea While Breathing Room Air

The speed of blood flow through the brain was greatly decreased and through the leg it was slightly increased.

The decrease in the flow of blood through the leg is not so easy to explain, for in Groups 7, 8 and 9, in which there had been a decrease either in the carbon dioxide or in the oxygen content of the arterial blood, the leg flow had increased. Throughout this investigation, the blood flow through the leg was more variable than the flow through the brain.

\section{$A-V$ differences in carbon dioxide content}

In the presentation of material thus far, we have dealt only with changes in oxygen tension of the blood. In Figures 1-10 the A-V dif- 
ferences in carbon dioxide content are shown in the right hand portion. One would expect that curves representing changes in oxygen and in carbon dioxide content would slope in opposite directions; e.g. the $\mathrm{A}-\mathrm{V}$ difference for carbon dioxide should increase as that for oxygen decreases. Of the nine experimental groups the slopes are opposite in five for the leg and in only one for the brain. As we have pointed out (12) the respiratory quotient (the increase in carbon dioxide of venous blood, divided by its decrease in oxygen with reference to the arterial blood) of the brain in

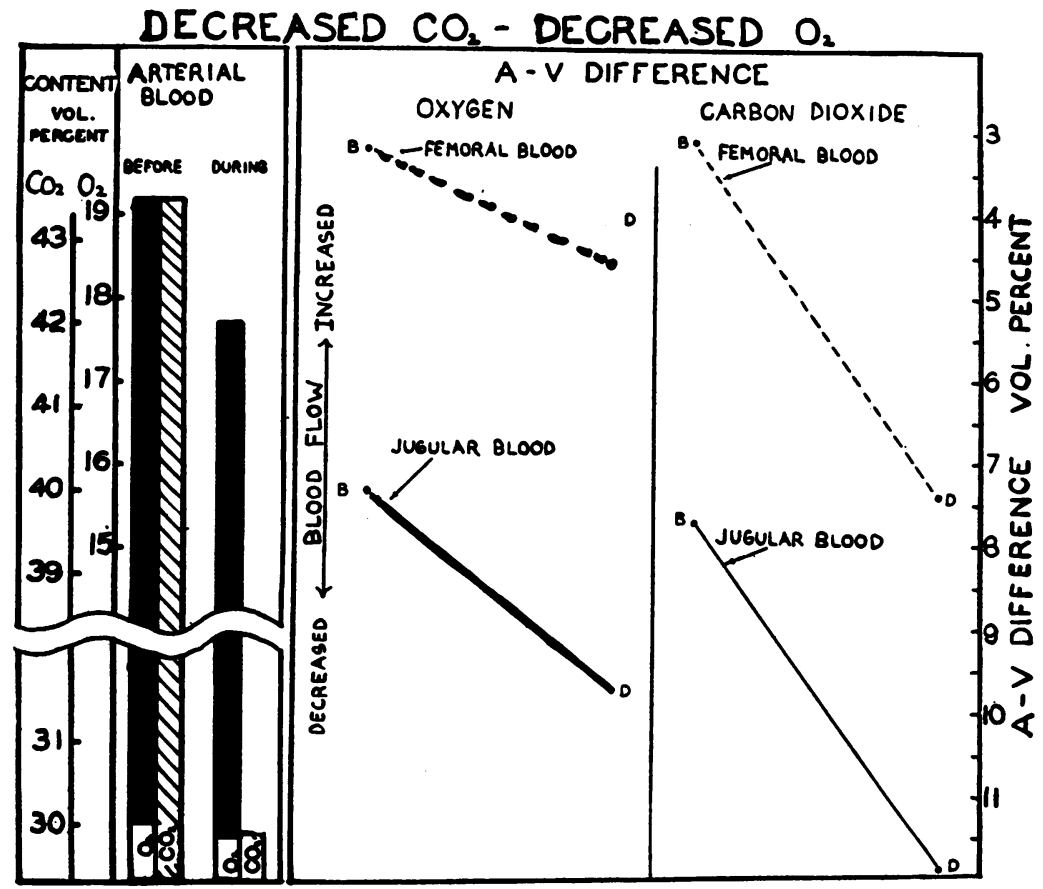

Fig. 10. Average Results in a Group of Five Subjects Who Performed Hyperpanea While Breathing Air Containing from 6 to 10 Per Cent OF OXYGen.

The speed of blood flow through both the brain and the leg was decreased.

these unanesthetized subjects is much higher than that of the leg. Under conditions in which the composition of the alveolar air is artificially altered the respiratory quotient is greatly distorted. In the low oxygen experiments one factor in such distortion is the various degrees of absorption of carbon dioxide by the tissues. In the ten experiments in which patients quietly breathed pure oxygen (Group 5) the average respiratory quotients before and after the procedure were approximately the same. In the seven experiments in which marked anoxemia was produced (Group 7), the quotients during the breathing were above unity, 1.32 for the brain and 1.80 for the leg, against preliminary averages of .91 


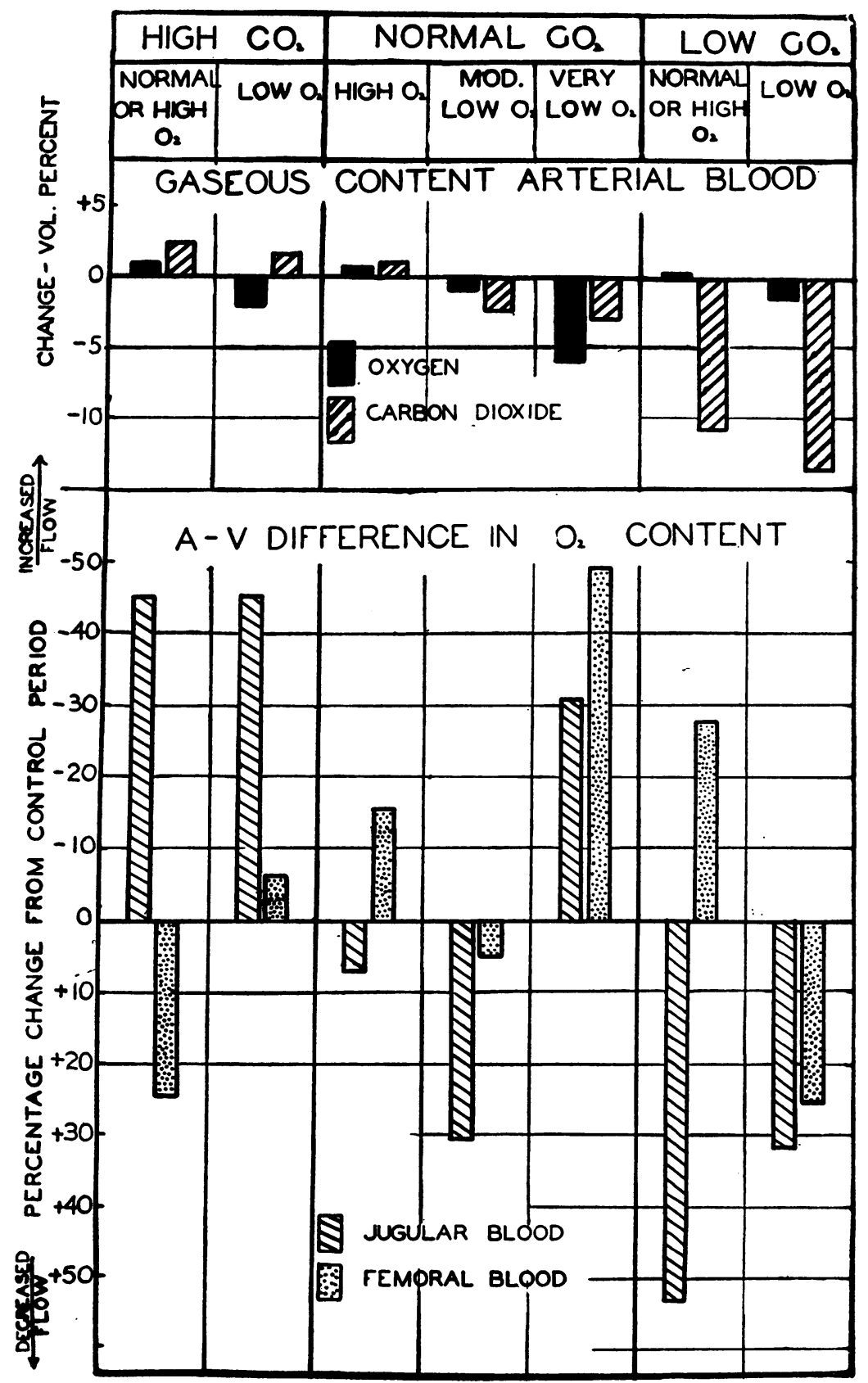

Fig. 11. The Average Results in Seven Groups of Experiments with 50 SuBJECTS

The upper portion of the figure represents the changes in the gaseous content of arterial blood which followed alteration of the alveolar air, induced by breathing various mixtures of carbon dioxide and oxygen. Projection of 
and .62 respectively. This excess of carbon dioxide in the venous blood suggests that under conditions of anoxemia carbon dioxide is not readily absorbed by the tissues, and more carbon dioxide is absorbed by the brain than by muscle. McGinty (13) found that tissues of living frogs do not absorb carbon dioxide as readily when the animals are kept in a low oxygen atmosphere and that the brain absorbed more than muscles. If the differences in respiratory quotient between the brain and the leg had been due to differences in the distribution of capillaries in the brain and leg (a possibility suggested by Myerson, Loman, Edwards and Dill (14) for similar respiratory quotients in their cases) one would expect distinct differences in blood flow. On the contrary the increase in the speed of blood flow in Group 7 was practically the same in the brain as in the leg.

\section{DISCUSSION}

The detailed changes in blood flow which have been enumerated are confusing. In order to contrast more clearly the average changes in each group, we calculated the percentage change in the A-V oxygen difference for the experimental as contrasted with the control period. These results are depicted in Figure 11.

At the top of the chart is shown the average change from the preexperimental sample as regards the gaseous content of arterial blood. The changes in the composition of blood flowing through the brain are clear cut and consistent. An increase in the carbon dioxide content of the arterial blood causes an increase of more than 40 per cent in blood flow, as measured by alterations in the oxygen content of venous blood. On the other hand, a similar decrease in the carbon dioxide of arterial blood results in a decrease in blood flow only a third or a fourth as great. Wolff and Lennox observed this also when watching pial blood vessels.

The effect of oxygen is less. An increase of oxygen in arterial blood causes a slight decrease in cerebral flow, a well marked anoxemia an increase. When the degree of anoxemia is not great, any change in the carbon dioxide tension of blood overshadows it. However, anoxemia partially neutralizes the constricting effect of diminished carbon dioxide. Also, anoxemia augments the dilator effect of carbon dioxide and excess oxygen augments the constrictor effect of decreased carbon dioxide.

These conclusions are in entire agreement with the window observations of Wolff and Lennox. Dilatation of pial vessels of cats occurred

columns above or below the base line indicates that the gaseous content had been increased or decreased. The solid columns indicate oxygen content, the double hatched columns carbon dioxide content. The lower portion of the figure represents changes which took place in the A-V difference of blood oxygen. Projection of columns above the base line indicates that the A-V difference had decreased and the speed of blood flow had increased. Projection below the line indicates the opposite. The single hatched columns represent the brain and the dotted columns the leg. 
under the same circumstances that cause decrease in the difference in the blood gases entering and leaving the brain. The effect of changes in oxygen tension of arterial blood was more clear cut in the human than in the cat experiments, due presumably to the fact that the anesthetized animals had a mild degree of anoxemia.

Our observations demonstrate that the changes in the diameter of pial vessels do not indicate, as Kubie and Hetler (15) suggested, merely a local change in pial circulation, but reflect, rather, a change in circulation throughout the brain. Likewise they contradict the contention of Nicholson (16) that the increase in cerebrospinal fluid flow which accompanies breathing of carbon dioxide is not due to enlargement of the cerebral vascular bed.

Concerning the effects of altering blood gases on the circulation of the leg, the evidence is less clear cut. Given a normal or better than normal arterial oxygen tension, an increased carbon dioxide tension was associated with an increased A-V difference (decrease in blood flow). A decreased carbon dioxide tension was associated with a decreased A-V difference (increase in blood flow). This is the opposite to the condition for the brain. In the presence of a profound arterial anoxemia (the carbon dioxide tension being only slightly affected) there is a markedly increased blood flow, through both leg and brain.

In general, it may be said that circulatory responses to chemical stimuli are greater in the brain than in the leg. One reason for this is the fact, pointed out by Lennox and Leonhardt (17), that the A-V difference is greater for the brain than for the extremities. Therefore there is greater opportunity for a decreased $\mathrm{A}-\mathrm{V}$ difference, in other words, for an improvement in the quality of the blood pouring through the brain. Cerebral circulatory responses are also more constant than those of the leg from time to time and from subject to subject.

These observations demonstrate that the body has a mechanism for altering the speed and the composition of the blood flowing through the brain. Either anoxemia or increase of carbon dioxide is attended by a dilatation of vessels, which provides capillaries and veins with blood that is more nearly arterial in quality. The opposite conditions result in a retardation of the blood stream. The chemical mechanism which governs these circulatory changes is probably a complicated one. In the case of the carbon dioxide, the coincident alterations in the acid base relationships of the blood are undoubtedly important. Injection of acid substances other than carbon dioxide or of alkaline substances also result in dilatation and constriction of brain vessels. Presumably the increased cerebral flow consequent on anoxemia cannot be explained on the basis of acid base changes in the blood, for according to Koehler, Brunquist and Loevenhart (18) acidosis occurs only when the anoxemia is extreme and prolonged. Whatever the exact mechanism, these adjustments in blood 
flow provide brain tissue with blood which is more constant in quality than would otherwise be the case. Presumably this contributes to more effective functioning of the brain.

The fact that circulatory adjustments for the brain may be and usually are the opposite of those for the leg is of great theoretical and practical interest. This circumstance permits alteration of blood flow in the brain without change in the systemic blood pressure. It permits the brain to secure preferential treatment as regards allocation of arterial blood. In these experiments the differential action is more evident after alteration of carbon dioxide than after alteration of oxygen tension. When the carbon dioxide tension of the arterial blood was increased or reduced (and oxygen tension not reduced) the speed of blood flow in the head and leg was affected in an opposite manner. On the other hand, when anoxemia was induced (no matter what the carbon dioxide tension) the blood flow in the brain and leg increased or decreased together. These are average, not individual results.

These experiments do not shed light on the localization of the mechanism of chemical control, whether in a center in the brain or in the walls of the blood vessels. One is perplexed to explain how the same chemical stimulus produces such diverse vasomotor effects. Nor can one explain the differing reactions on the basis of the closed box arrangements of the skull. Though vessels in the brain are embedded in an incompressible medium, the alterations in calibre, as measured by A-V differences, are even greater in the brain than in the leg. Of course, one must take into account possible differences in the area of the vascular bed in relation to the volume of brain and leg tissues. According to the researches of Cobb (19) nervous tissue has fewer capillaries per square millimeter than has muscle. Again, capillaries in the two regions may respond differently to chemical stimuli, or there may be differing responses of capillaries and of arterioles. The relative scarcity of capillaries in the brain, together with the external resistance to capillary expansion, may hinder capillary stasis and hence, when arterioles dilate, result in a relatively speedy flow of blood through the brain.

Finally our observations emphasize the contention of Wolff and Lennox (2) that when the patient benefits from measures which alter the composition of alveolar air, in any assignment of credit, the accompanying changes in cerebral circulation must be taken into account.

\section{SUMMARY}

Fifty observations were made of changes in the speed of blood flow through the brain and the leg of man. These changes were in response to alteration in the composition of the alveolar air, and were judged by the alterations in the difference in the oxygen content of blood entering and leaving the brain and the leg. 
Alteration in the composition of alveolar air was attended by a marked change in the speed of blood flow. With an increased tension of carbon dioxide in arterial blood, the speed of flow through the brain was increased and through the leg was decreased. With a decreased tension of carbon dioxide the flow through the brain was decreased and through the leg increased.

Alteration of the oxygen content of arterial blood produced less pronounced results. Increase in the oxygen content resulted in a slight decrease in speed of blood flow through the brain and increase through the leg. Pronounced anoxemia resulted in increased flow in both brain and leg.

Changes in speed of flow in these experiments are believed to be due to dilatation or constriction of arterioles.

An increase in the flow in the brain is more readily produced than a decrease. Alterations in the flow are more consistent in the brain than in the leg. Conclusions are based on average, not individual, results.

In conditions of anoxemia the tissues of the brain and the leg absorb less than the usual amount of carbon dioxide.

Circulatory adjustments for the brain, in response to gaseous changes in the blood, may be the opposite for those in the leg, thereby permitting the brain to receive preferential treatment as regards allocation of arterial blood.

\section{BIBLIOGRAPHY}

1. Forbes, H. S. and Wolff, H. G., Arch. Neurol. and Psychiat., 1928, xix, 1057. Cerebral Circulation. III. The Vasomotor Control of Cerebral Vessels.

2. Wolff, H. G. and Lennox, W. G., Arch. Neurol. and Psychiat., 1930, xxiii, 1097. Cerebral Circulation. XII. The Effect on Pial Vessels of Variations in the Oxygen and Carbon Dioxide Content of the Blood.

3. Myerson, A., Halloran, R. D. and Hirsch, H. L., Arch. Neurol. and Psychiat., 1927, xvii, 807. Technic for Obtaining Blood from the Internal Jugular Vein and Internal Carotid Artery.

4. Bronk, D. W. and Gessell, R., Am. J. Physiol., 1927, lxxxii, 170. The Regulation of Respiration. X. Effects of Carbon Dioxide, Sodium Bicarbonate, and Sodium Carbonate on the Carotid and Femoral Flow of Blood.

5. Van Slyke, D. D., J. Biol. Chem. 1927, lxxiii, 121. Notes on a Portable Form of the Manometric Gas Apparatus and on Certain Points in the Technique of Its Use.

6. Weiss, S. and Lennox, W. G., Arch. Neurol. and Psychiat., 1931, xxvi, 737. The Cerebral Circulation. XVII. Cerebral Blood Flow and the Vasomotor Response of the Minute Vessels of the Human Brain to Histamine.

7. Cobb, S. and Fremont-Smith, F., Arch. Neurol. and Psychiat., 1931, xxvi, 731. The Cerebral Circulation. XVI. Changes in the Human Retinal Circulation and the Pressure of the Cerebrospinal Fluid during Inhalation of a Mixture of Carbon Dioxide and Oxygen. 
8. Lennox, W. G., Certain Chemical and Physiological Conditions Which May Influence Seizures. Epilepsy and the Convulsive States. Chapter XV, p. 471. Williams and Wilkins Company, Baltimore, 1931.

9. Bernthal, T. G., Bronk, D. W., Cordero, N. and Gesell, R., Am. J. Physiol., 1928, 1xxxiii, 435. The Regulation of Respiration. XVII. The Effects of Low and High Alveolar Oxygen Pressure and of Sodium Cyanide on the Carotid and Femoral Flow of Blood as Studied with the Continuous Electrometric Method.

10. Marshall, E. K., Jr., Medicine, 1930, ix, 175. The Cardiac Output of Man.

11. Schneider, E. C. and Clarke, R. W., Am. J. Physiol., 1929, lxxxviii, 633. Studies of Muscular Exercise under Low Barometric Pressure. IV. The Pulse Rate, Arterial Blood Pressure and Oxygen Pulse.

12. Lennox, W. G. and Leonhardt, E., Arch. Neurol. and Psychiat., 1931, xxvi, 719. The Cerebral Circulation. XIV. The Respiratory Quotient of the Brain and of the Extremities in Man.

13. McGinty, D. A., Am. J. Physiol., 1930, xciii, 528. The Regulation of Respiration. XXXIV. The Carbon Dioxide Content of the Intact Brain of the Frog in Relation to Changes of Oxidations.

14. Myerson, A., Loman, J., Edwards, H. T., and Dill, D. B., Am. J. Physiol., 1931, xcviii, 373. The Composition of Blood in the Artery, in the Internal Jugular Vein and in the Femoral Vein during Oxygen Want.

15. Kubie, L. S. and Hetler, D. M., Arch. Neurol. and Psychiat., 1929, xx, 749. The Cerebral Circulation. IV. A Study of the Circulation in the Cortex by Means of Color Photography.

16. Nicholson, H., Am. J. Physiol., 1932, xcix, 570. Effects of Low Alveolar Oxygen and High Alveolar Carbon Dioxide on the Rate of Flow of Cerebrospinal Fluid.

17. Lennox, W. G. and Leonhardt, E., Arch. Int. Med., 1930, xlvi, 630. The Oxygen and Carbon Dioxide Content of Blood from the Internal Jugular and Other Veins.

18. Koehler, A. E., Brunquist, E. H., and Loevenhart, A. S., J. Biol. Chem. 1925, lxiv, 313. The Production of Acidosis by Anoxemia.

19. Cobb, S., Ârch. Surg., 1929, xviii, 1200. The Cerebral Circulation. VIII. A Quantitative Study of the Capillaries in the Hippocampus. 